\title{
Estudo comparativo entre fentanil e sufentanil sobre a pressão intraocular em cães anestesiados com halotano
}

\section{Comparative study between sufentanil and fentanyl on intraocular pressure in dogs anesthetized with halothane}

\author{
Eunice Yuriko Tamura, ${ }^{*}$ Paulo Sergio de Moraes Barros, ${ }^{\star *}$ Denise Tabacchi Fantoni, ${ }^{\star \star *}$ \\ Silvia Renata Gaido Cortopassi $i^{\star * * *}$
}

\begin{abstract}
Resumo
Neste estudo comparou-se a ação do fentanil e sufentanil sobre a pressão intraocular (PIO) no período transoperatório. Para tanto foram utilizados 14 cães de diferentes raças e idades, com peso variando entre 10 e $15 \mathrm{~kg}$ e submetidos a protocolos cirúrgicos ortopédicos. Os animais foram pré-tratados com acepromazina e a indução anestésica foi realizada com tiopental sódico. Após intubação orotraqueal, receberam halotano em oxigênio a $100 \%$ e após adequada estabilização, o bloqueador neuromuscular (pancurônio). Imediatamente antes do início do procedimento cirúrgico, os animais foram distribuídos aleatoriamente em dois grupos: os animais do grupo I receberam fentanil e os animais do grupo II o sufentanil. Foram avaliadas a freqüência cardíaca, pressão arterial sistêmica não invasiva e a pressão intraocular. A concentração do agente anestésico inalatório no ar inspirado foi mantida constante durante todo o procedimento através de analisador de gases. Pudemos observar que houve um decréscimo dos valores de freqüência cardíaca nos valores do grupo Il após os momentos posteriores ao da aplicação do opóide. Na comparação entre os grupos observou-se que os animais do grupo II apresentaram valores inferiores de freqüência cardíaca aos 5 e 10 minutos após a aplicação do opióide. Não foi observada diferença estatística entre os valores de pressão arterial e intraocular em nenhum dos grupos ou na comparação entre os grupos. Através da análise dos resultados pôde-se concluir que o fentanil não promove qualquer alteração sobre a PIO sendo um agente indicado para a manutenção da anestesia de cães submetidos a procedimentos cirúrgicos oftálmicos; já o sufentanil, mesmo não promovendo importante alteração da PIO, é um agente que deve ser utilizado com cautela na manutenção da anestesia, uma vez que provoca moderada bradicardia.
\end{abstract}

Palavras-chave: pressão intraocular; fentanil; sufentanil; cães.

\section{Introdução}

Com o desenvolvimento da oftalmologia em Medicina Veterinária faz-se necessário um aprimoramento das técnicas anestésicas para cirurgias oftálmicas. Os procedimentos cirúrgicos intraoculares necessitam de adequado plano anestésico, assim como da centralização do globo ocular e da manutenção estável da pressão intraocular (PIO) nos limites fisiológicos do animal em questão. Desta forma, têm-se estudado as diferentes técnicas anestésicas, buscando aquela que melhor atue nos procedimentos intraoculares, prevenindo a elevação ou diminuindo os valores da PIO.

A pressão intraocular é definida como a pressão exercida pelo conteúdo dos olhos sobre a parede que o contém. Esta pressão é determinada pelo volume de vários componentes internos do olho, como humor aquoso e sangue (os quais exercem uma pressão centrífuga), e por forças compressivas externas (Murphy, 1985).

Os opióides são, dentre os analgésicos conhecidos, os mais potentes (Short, 1987). Seu uso na pré-anestesia, no trans e pós-operatório é muito difundido em cães (Short, 1987; Nolan \& Reid. 1991; Wilson, 1992); a maior razão para utilização destas drogas é a mínima alteração cardíaca que produzem (Pascoe, 1992).

O fentanil é um opióide primariamente agonista, 80 vezes mais potente que a morfina; seus efeitos analgésicos e euforizantes são antagonizados pelos antagonistas de receptores opióides como a naloxona. A analgesia, sedação e depressão respiratória ocorrem após quatro minutos da administração intravenosa com um pico de ação dentro de 10 a 15 minutos, e tempo efetivo de 30 minutos (Soma \& Shields, 1964).

\footnotetext{
*Bolsista de aperfeiçoamento CNPq - Departamento de Cirurgia da Faculdade de Medicina Veterinária e Zootecnia da Universidade de São Paulo.

** Professor Titular do Departamento de Cirurgia da Faculdade de Medicina Veterinária e Zootecnia da Universidade de São Paulo.

*** Professor Assistente do Departamento de Cirurgia da Faculdade de Medicina Veterinária e Zootecnia da Universidade de São Paulo.

${ }^{\star \star \star \star}$ Pós-graduanda do Departamento de Cirurgia da Faculdade de Medicina Veterinária e Zootecnia da Universidade de São Paulo.
} 
O sufentanil é um análogo do fentanil de ação mais curta, maior potência (aproximadamente 10 vezes) o que implica doses menores para atingir a mesma resposta clínica que o fentanil (Murphy, 1993). Foi comprovado, após experimentos em ratos, que o sufentanil foi o analgésico mais completo, quando comparado a outros sete morfinomiméticos, pois promoveu redução mais importante da concentração alveolar mínima (Hecker et. al.; 1985). Em estudos realizados com cães, utilizando-se altas doses de sufentanil, verificou-se sutil bradicardia e pequena queda da pressão sistólica e diastólica (De Castro et al., 1979; Eriksen et al., 1981).

A mensuração da $\mathrm{PIO}$ em cães pode ser realizada por meio de tonômetro de aplanação, o qual mede a força necessária para aplanar uma área constante da superfície central da córnea. Na avaliação comparativa entre diferentes tonômetros (Mackay-Marg, Tono-pen e Challenger), a Tono-pen apresentou vantagens significativas em relação aos outros, no que se refere à precisão, tamanho, portabilidade e versatilidade em oftalmologia veterinária (Priehs et al., 1990).

Através deste experimento objetivou-se avaliar comparativamente a ação do fentanil e do sufentanil sobre a PIO de cães, com o intuito de obtermos novas técnicas isentas de efeitos adversos para a realização de cirurgias oftálmicas nesta espécie; para tanto, analisou-se também a pressão arterial e freqüência cardíaca. Além disso avaliou-se a ação dos opióides frente a procedimentos oftálmicos, podendo ser uma droga importante para a manutenção da anestesia no transoperatório de cirurgias intraoculares.

\section{Material e método}

Foram utilizados 14 cães adultos de diferentes raças, com peso variando entre 10 e $15 \mathrm{~kg}$, atendidos no Hospital Veterinário da Faculdade de Medicina Veterinária e Zootecnia da Universidade de São Paulo. Os animais foram submetidos previamente a jejum hídrico de quatro horas e alimentar de 12 horas. Foram realizados procedimentos cirúrgicos ortopédicos, osteossínteses, em todos os animais.

Os animais foram distribuídos aleatoriamente em dois grupos: grupo I e grupo II. Todos os animais receberam $0,1 \mathrm{mg} /$ $\mathrm{kg}$ de peso vivo (PV) de acepromazina ${ }^{1}$ pela via intravenosa (IV). Decorridos 10 minutos da aplicação da acepromazina, foram administrados $12,5 \mathrm{mg} / \mathrm{kg}$ de tiopental ${ }^{2}$ pela via intravenosa. Uma vez que os animais apresentaram relaxamento da mandíbula e ausência do reflexo laringotraqueal, foram intubados com sonda de diâmetro adequado, e esta foi conectada ao circuito circular fechado do aparelho de anestesia. ${ }^{3}$ A manutenção anestésica foi realizada com auxílio do halotano ${ }^{4}$ em oxigênio a $100 \%$, mantendo-se a concentração do agente no ar expirado entre $0,4 \%$ e $0,6 \%$, através de um analisador de gases. ${ }^{5}$ Administrou-se então o pancurônio ${ }^{6}$ na dose de $0,06 \mathrm{mg} / \mathrm{kg}$ pela via intravenosa. Os animais foram mantidos sob ventilação controlada com freqüência respiratória de 12 movimentos por minuto e volume corrente de $15 \mathrm{ml} / \mathrm{kg}$. Um minuto antes da incisão da pele, os animais do grupo I receberam fentanil 7 na dose de $5 \mathrm{mg} / \mathrm{kg}$ pela via IV e os animais do grupo II sufentanil${ }^{8}$ na dose de $1 \mathrm{mg} / \mathrm{kg}$ pela via IV. Nos animais onde o tempo cirúrgico ultrapassou o tempo de avaliação deste experimento, doses complementares de opióides foram aplicadas de acordo com a necessidade do paciente.

No decorrer do experimento foram avaliados os seguintes parâmetros: pressão intraocular (PIO), pressão arterial sistólica (PAS), média (PAM), diastólica (PAD) e freqüência cardíaca (FC).

Estas variáveis foram avaliadas da seguinte maneira:

a) Pressão intraocular: mensurada com tonômetro de aplanação. ${ }^{9}$ Após a anestesia da córnea com colírio anestésico, ${ }^{10}$ a ponteira foi colocada sobre a superfície da córnea e três medidas da PIO foram coletadas para cada momento.

b) Pressão arterial: avaliada através de mensuração indireta com a utilização de monitor de pressão não invasivo, ${ }^{11}$ sendo o manguito colocado no membro anterior, com o animal em decúbito lateral, obtendo desta forma a pressão sistólica, média e diastólica.

c) Freqüência cardíaca: avaliada através de monitor cardíaco. $^{12}$

Todos os parâmetros foram avaliados nos seguintes tempos: três minutos após a aplicação do bloqueador neuromuscular e um, três, cinco e 10 minutos após a aplicação do opióide.

Posteriormente, os valores obtidos nos dois grupos foram submetidos à análise estatística através de provas paramétricas - análise de variância seguida do teste de Tukey entre os valores obtidos nos diferentes momentos de avaliação, do mesmo grupo, e análise comparativa através do teste T de Student entre os valores obtidos no mesmo momento entre os dois grupos. O grau de significância estabelecido foi de $5 \%(p<0,05)$.

\section{Resultados}

Os resultados obtidos estão apresentados na Tabela 1.

Em relação à freqüência cardíaca, observou-se decréscimo dos valores no grupo II após a aplicação do opióide, sendo esta diferença estatisticamente significativa em to-

\footnotetext{
${ }^{1}$ Acepran a 0,2\% - Univet S.A.; ${ }^{2}$ Thionembutal - Abbott Lab. do Brasil Ltda.; ${ }^{3}$ Takaoka - Samurai III; ${ }^{4}$ Fluothane - Lab. Wellcome - Zeneca Ltda.; ${ }^{5}$ Analisador de gases - Multinex / Datascope.; ${ }^{6}$ Pavulon - Akzo Nobel Ltda.; ${ }^{7}$ Fentanil - Janssen; ${ }^{8}$ Sufentanil - Janssen; ${ }^{9}$ Tono-Pen -

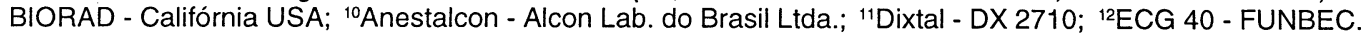




\begin{tabular}{|c|c|c|c|c|c|c|}
\hline \multirow[b]{2}{*}{ Parâmetros } & \multirow[b]{2}{*}{$\begin{array}{c}\text { Grupos } \\
n=14\end{array}$} & \multirow[b]{2}{*}{$\begin{array}{c}3 \text { min após } \\
\text { BNM }\end{array}$} & \multicolumn{4}{|c|}{ Minutos após administração do opióide } \\
\hline & & & 1 & 5 & 10 & 15 \\
\hline FC & Gl: & $143.3 \pm 29$ & $119.6 \pm 49$ & $127.3 \pm 37 \dagger$ & $114.6 \pm 28 \dagger$ & $115.6 \pm 21$ \\
\hline (bat/min) & Gll & 150 & $88.4 \pm 27^{*}$ & $90.6 \pm 28^{*} \dagger$ & $84.7 \pm 34^{*} \dagger$ & $99.1 \pm 46^{*}$ \\
\hline PAS & $\mathrm{Gl}$ & $120.1 \pm 16$ & $118.4 \pm 16$ & $123.3 \pm 14$ & $136.3 \pm 27$ & $119.7 \pm 27$ \\
\hline$(\mathrm{mmHg})$ & Gll & $123.0 \pm 16$ & $111.6 \pm 18$ & $113.1 \pm 24$ & $114.0 \pm 28$ & $110.7 \pm 22$ \\
\hline PAM & Gl & $90.4 \pm 18$ & $89.6 \pm 22$ & $90.7 \pm 20$ & $106.6 \pm 21$ & $95.6 \pm 22$ \\
\hline$(\mathrm{mmHg})$ & Gll & $90.7 \pm 21$ & $84.3 \pm 17$ & $85.3 \pm 19$ & $86.0 \pm 24$ & $84.7 \pm 24$ \\
\hline PAD & Gl & $75.4 \pm 21$ & $73.1 \pm 26$ & $71.5 \pm 26$ & $89.8 \pm 24 \dagger$ & $81.0 \pm 19$ \\
\hline$(\mathrm{mmHg})$ & Gll & $75.1 \pm 17$ & $64.8 \pm 17$ & $64.8 \pm 17$ & $64.7 \pm 18 \dagger$ & $68.8 \pm 20$ \\
\hline $\mathrm{PIO}$ & $\mathrm{Gl}$ & $12.4 \pm 4$ & $10.8 \pm 4$ & $11.1 \pm 4$ & $11.3 \pm 4$ & $11.7 \pm 4$ \\
\hline$(\mathrm{mmHg})$ & Gll & $11.0 \pm 3$ & $11.7 \pm 4$ & $10.6 \pm 3$ & $11.0 \pm 3$ & $10.0 \pm 3$ \\
\hline
\end{tabular}

Tabela 1: Valores médios e desvios-padrão da freqüência cardíaca, pressão arterial sistólica, média e diastólica e pressão intraocular de cães tratados com acepromazina, tiopental, pancurônio, fentanil e halotano (grupo I) e acepromazina, tiopental, pancurônio sufentanil e halotano (grupo II) em diferentes momentos de avaliação.

FC - freqüência cardíaca; PAS - pressão arterial sistólica; PAM - pressão arterial média; PAD - pressão arterial diastólica; PIO - pressão intraocular; BNM - bloqueador neuromuscular; bat/min - batimentos por minuto. *diferenças significantes em relação; ao valor basal; †diferença significante entre os grupos.

dos os momentos posteriores (um, cinco, dez e 15 minutos após a aplicação do opióide) quando comparados ao momento inicial (após a aplicação do bloqueador neuromuscular), sendo os valores de $\mathrm{p}$ respectivamente $p<0,01 ; p<0,01 ; p<0,01 ; p<0,01$ e $p<0,05$. Na comparação entre os grupos, os animais do grupo II apresentaram valores inferiores de FC aos cinco e 10 minutos após a aplicação do opióide, sendo a diferença pouco significante $(p=0,25)$.

Não foi observada diferença estatística entre os valores das pressões arterial sistólica e média em nenhum dos grupos ou na comparação entre os grupos.

Os valores de pressão arterial diastólica dos animais do grupo Il foram inferiores aos valores obtidos no grupo I aos 10 minutos após a aplicação do opióide, sendo a diferença estatisticamente significante.

Em relação à PIO não foi constatada nenhuma alteração significativa na avaliação entre momentos de mesmo grupo e entre os grupos.

\section{Discussão}

Em estudos realizados com cães, utilizando-se doses altas de sufentanil, verificou-se bradicardia moderada e pequena queda na pressão sistólica e diastólica (De.Castro et al., 1979; Eriksen et al., 1981): Da mesma forma, neste experimento observoutse que a ação do sufentanil no grupo ll levou à queda significativa da FC nos momentos que se sucederam a sua aplicação, sendo esta bradicardia revertida após 15 minutos, de acordo com o tempo de ação da droga Em relação à pressão arterial não foi observada nenhuma alteração. No grupo I, o fentanil não provocou nenhựma alteração significativa no sistema cardiovașcular; observou-se apenas uma bradicardia leve, estatisticamente não significante. O fentanil pode causar comportamento agressivo no pós-operatório; apnéia, salivação, bradicardia e relaxamento do esfíncter anal, com ocasional defecação. Estas alterações autonômicas ocorrem devido ao aumen- to do tônus parassimpático, e podem ser prevenidas pela administração prévia de sulfato de atropina (Soma \& Shields, 1964; Lumb \& Jones, 1996). Em nosso estudo estas variações não foram observadas.

Devido a alta potência do sufentanil (aproximadamente 10 vezes maior que a do fentanil), pequena quantidade da droga é necessária para atingir respostas clínicas vistas com doses efetivas de fentanil ( Murphy, 1993). Comparandose os parâmetros de FC entre os dois grupos verificou-se que o sufentanil apresentou um decréscimo ligeiramente maior que o fentanil, não significativo estatisticamente; este resultado é esperado uma vez que o sufentanil tem potência superior ao fentanil.

Em relação à $\mathrm{PIO}$, não foi verificado aumento deste parâmetro em nenhum dos momentos avaliados nos dois grupos experimentais, mantendo-se estável com ligeiros decréscimos não significativos.

O uso destes analgésicos opióides reduzem consideravelmente a concentração alveolar mínima de halotano, diminuindo os efeitos deletérios desta droga, como a hipotensão arterial que produz. Analisando-se o estudo aqui apresentado e a literatura consultada pode-se perceber que ainda permanecem dúvidas a respeito da ação dos analgésicos opióides em procedimentos de maior duração quando comparados ao usso do halotano na manutenção anestésica tanto em relação à PIO como também nas funções vitais do paciente.

A partir deste estudo foi possível concluir que o fentanil não promove qualquer alteração sobre a PIO e não interfere no sistema cardiovascular de modo deletério, podendo șer utilizado na manutenção anestésica em procedimentos cirúrgicos oftálmicos; e também que o sufentanil não promove qualquer alteração sobre a PIO, promovendo até um decréscimo desta, porém por provocar bradicardia moderada esta droga deve ser empregada com cautela em pacientes que apresentem alterações prévias do sistema cardiovascular. 


\section{Abstract}

In this study we evaluated comparatively the effect of fentanyl and sufentanil on the intraocular pressure in the dogs during the orthopaedic surgery. Fourteen dogs of different breeds and ages, weight ranging from 10 to $15 \mathrm{~kg}$ were randomly assigned to two groups : animals of group one received the association of acepromazine/ thiopental/ halothane/ pancuronium/ fentanyl and the animals of group two received the same pre anesthesic medication/ drug induction/ halothane/ pancuronium/ sufentanil. Heart hate, arterial pressure and intraocular pressure were evaluated in different times. Statistical analysis showed that the use of fentanyl did not promote any change on intraocular pressure, and so did sufentanil that could sometimes decrease this pressure, but promoted bradicardia and must be used with caution.

Keywords: intraocular pressure; fentanyl; sufentanil; dogs.

\section{Referências bibliográficas}

De CASTRO, J., Van De WATER, A., WOUTERS, L., XHONNEUX, R., RENEMAN, R., KAY, B. Comparative study of cardiovascular, neurological and metabolic side-effects of eight narcotics in dogs: pethidine, piritramide, morphine, phenoperidine, fentanyl, R 39 209, sufentanil R 34995. Acta Anaesthesiology. Belg. v. 30, p. 5, 1979.

ERIKSEN, J. , BERTHELSEN, P., AHN, N.C., RASMUSSEN, J.P. Early response in central hemodynamics to high doses of sufentanil or morphine in dogs. Acta Anaesthesiologica Scandinavica. V. 25, p. 33, 1981.

HECKER, B. R. , LAKE, C. L., DIFAZIO, C. A. et al. The reduction in halothane MAC with sufentanil. Anesthesiology v. 59, p. 341, 1985.

LUMB, W. V., JONES, E. W. Veterinary anesthesia. $2^{\text {nd }}$ ed. Philadelphia, Lea \& Febiger, 1996, p.190

MURPHY, D.F. Review article: Anesthesia and intraocular pressure. Anesthesia and Analgesia, v. 64: p. 520-530, 1985.

MURPHY, M. R. Opióides in BARASH ;P.G., CULLEN,B.F., STOELTING,R.K. Tratado de anestesiologia clínica. v. 2, 1. ed. cap. 9. Rio de Janeiro: Manole, 1993, p. 331.
NOLAN, A. M., REID, J. The use of intraoperative fentanyl en spontaneously breathing dogs undergoing orthopaedic surgery. Journal of Veterinary Anesthesia, v. 18, p. 30-34, 1991.

PASCOE, P.J. The case of maintence of genenral anaesthesia with a injectable agent. The Veterinary Clinics of Nothe America - Small Animal Practice, v. 22, n. 2, p. 275-277, 1992.

PRIEHS,D.R., GUM,G.G., WHITLEY,D., MOORE,L.E. Evaluation of three applanation tonometers in dogs. American Journal of Veterinary Research, v. 51, n. 10, p.1547, 1990.

SHORT, C. E. Principles \& pratice of veterinary anesthesia. Los Angeles, Williams \& Wilkins, 1987, p. 669.

SOMA, L.R., SHIELDS, D. R. Neuroleptoanalgesia produced by fentanyl and droperidol. Journal of American Veterinary Medicine Association, v. 145, n. 9, p. 897902, 1964.

WILSON, D. V. Advantages and guidelines for using opioid agonists for induction of anesthesia. The Veterinary Clinics of North America - Small animal pratice, v. 22, n. 2, p. $269-$ 272, 1992. 